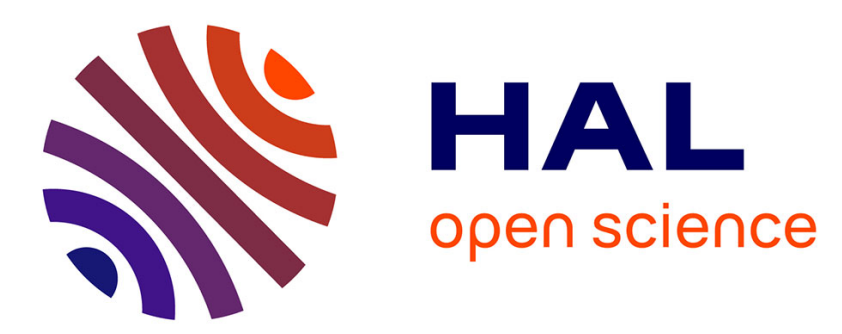

\title{
Time resolved nonlinear spectroscopy at the band edge of 1D photonic crystals
}

Magali Astic, Philippe Delaye, Robert Frey, Gérald Roosen, Régis André, N. Belabas, Isabelle Sagnes, R. Raj

\section{> To cite this version:}

Magali Astic, Philippe Delaye, Robert Frey, Gérald Roosen, Régis André, et al.. Time resolved nonlinear spectroscopy at the band edge of 1D photonic crystals. Journal of Physics D: Applied Physics, 2008, 41 (22), pp.224005. 10.1088/0022-3727/41/22/224005 . hal-00554084

\section{HAL Id: hal-00554084 \\ https://hal-iogs.archives-ouvertes.fr/hal-00554084}

Submitted on 10 Jan 2011

HAL is a multi-disciplinary open access archive for the deposit and dissemination of scientific research documents, whether they are published or not. The documents may come from teaching and research institutions in France or abroad, or from public or private research centers.
L'archive ouverte pluridisciplinaire HAL, est destinée au dépôt et à la diffusion de documents scientifiques de niveau recherche, publiés ou non, émanant des établissements d'enseignement et de recherche français ou étrangers, des laboratoires publics ou privés. 


\title{
Time resolved nonlinear spectroscopy at the band edge of $1 \mathrm{D}$ photonic
}

\section{crystals}

\author{
M. Astic, Ph. Delaye, R. Frey, and G. Roosen \\ Laboratoire Charles Fabry de l'Institut d'Optique, Centre National de la Recherche \\ Scientifique et Université Paris-Sud, \\ Campus Polytechnique, RD 128, 91128 Palaiseau cedex, France
}

\section{R. André}

CEA-CNRS group « Nanophysique et semiconducteurs 》

Institut NEEL-CNRS, BP 166, 38042 Grenoble cedex, France

N. Belabas, I. Sagnes, R. Raj

Laboratoire de Photonique et Nanostructures, Centre National de la Recherche Scientifique, Site Alcatel de Marcoussis, Route de Nozay, 91460 Marcoussis, France

\begin{abstract}
Large refractive index changes have been measured at the band edge frequency of $1 \mathrm{D}$ photonic crystals. Results concerning both thin and thick samples of high and low refractive index contrast respectively are presented. The very large value of the refractive index changes obtained at moderate pump powers thanks to the strong enhancement of the local intensity inside the photonic crystal open the way to very small volume devices for optical signal processing. However, time-resolved experiments demonstrate the photo-generation of high free carrier densities through two- or even three-photon absorptions which are shown to be also strongly enhanced at the band edge of the photonic crystal. This drawback may the most probably be circumvented by using lower pump intensities in photonic crystals showing narrower resonances.
\end{abstract}




\section{Introduction}

Due to their original light propagation properties, photonic crystal (PC) structures allow to realize high quality and small dimension devices, such as waveguides, delay lines, couplers or mirrors, opening the way to the realization of the future compact optical microcircuits $[1,2]$ for all optical classical and quantum signal processing. These properties are also very beneficial to favour nonlinear interactions. For instance, the large dispersion observed at the band edge can be used to control phase matching in second and third order wavelength conversion mechanisms [3-7]. Even the photonic band gap that prevents light to propagate inside the structure can be used to realize new waveguide propagation mechanisms that can favour nonlinearities [8,9]. Finally, photonic crystals are mainly used to enhance the efficiency of nonlinear interactions. Although such enhancements were already observed in 2D PC [10], for the sake of simplicity concerning the comparison with theory, the paper deals with simple 1D devices. For example slow waves and light localization at the band edge or on a defect of the photonic crystal increases the optical field amplitude in the structures inducing thus an enhancement of the effective nonlinear susceptibilities of the structure [11-17]. All these studies open the way to the realization of compact and rapid optimized nonlinear devices, once the interaction between the nonlinear mechanisms of different origins and the photonic crystal structure will be well characterized and understood.

Here, we present a study concerning the enhancement of optical third order nonlinear susceptibilities in 1D photonic crystals. The results were obtained through the measurement of the refractive index changes photo-induced at the wavelength of the band edge of the 1D photonic crystal. The two cases of low refractive index contrast large period number photonic crystals and high contrast photonic crystals composed of only ten periods are considered and the potential interest of such structures for the purpose of nonlinear optical signal processing is demonstrated. 
Section I is devoted to the description of the principle used for the measurement of the refractive index changes and the experimental setup used for this purpose. Section II shows and discusses nonlinear reflexion spectra recorded around the band edge of both CdMnTe/CdMgTe and GaAlAs/AlOx photonic crystals. Section III presents time resolved experiments allowing the description of the physical origin of the photo-induced refractive index changes in both photonic crystals considered in section II. Finally, section IV compares results obtained for the two photonic crystals and gives some insights for the future realization of nonlinear devices for compact optical signal processing.

\section{Principle and Experimental Setup}

\section{Theory}

The determination of the refractive index change is performed through the careful experimental analysis of the wavelength shift of the intensity dependent reflectivity spectrum of the photonic crystal that occurs at a band edge of the photonic crystal. In the case of 1D photonic crystal this is made very simply. Indeed, as verified using the numerical formalism reported in reference 12 , the band edge wavelength does not depend on losses present in the semiconductor materials of the 1D-PC, and an analytical analysis in a lossless photonic crystal is possible. In such a case, for a stack of $N$ pairs composed of two layers of thicknesses $e_{1}$ and $e_{2}$ with refractive indices $n_{1}$ and $n_{2}$ respectively, the transmission $T_{N}$ is given by [17]

$$
\frac{1}{T_{N}}=1+\frac{\sin ^{2} N \beta}{\sin ^{2} \beta}\left[\frac{1}{T}-1\right]
$$

Where $\beta$ is the Bloch phase given by

$$
\cos (\beta)=\operatorname{Re}\left\{\frac{1}{t}\right\}
$$


with $\mathrm{t}$ and $\mathrm{T}=|\mathrm{t}|^{2}$ the amplitude and intensity transmission coefficients of a pair respectively. For $\lambda / 4$ optical path layers $\left(n_{1} e_{1}=n_{2} e_{2}=\lambda_{0} / 4\right)$ the Bloch phase is related to the wavelength $\lambda$ through the relation

$$
\cos (\beta)=\frac{\cos (\pi \tilde{\lambda})-R_{12}}{T_{12}}
$$

Where $\tilde{\lambda}=\lambda_{0} / \lambda$ is the normalized wavelength with $\lambda_{0}$ the central wavelength of the forbidden band. In equation I.3 $T_{12}=4 n_{1} n_{2} /\left(n_{1}+n_{2}\right)^{2}$ and $R_{12}=\left(n_{1}-n_{2}\right)^{2} /\left(n_{1}+n_{2}\right)^{2}$ stand for the transmission and reflexion coefficients of interfaces respectively $\left(R_{12}+T_{12}=1\right)$. The band edge which corresponds to the first maximum of transmission $T_{N}$ is obtained for $\beta=\frac{N-1}{N} \pi$ [18]. The wavelength $\lambda_{B}$ of the band edge is then given by

$$
\cos \left(\pi \frac{\lambda_{0}}{\lambda_{B}}\right)=-\cos \left(\frac{\pi}{N}\right) T_{12}+R_{12}
$$

At high intensities, due to the modification of the refractive indices $\left(n_{i}^{\prime}=n_{i}+\Delta n_{i}\right.$, with $\Delta n_{i}$ the index changes $(i=1,2))$, the transmission and reflexion coefficients of the interfaces become $T_{12}^{\prime}=T_{12}+\Delta T_{12}$ and $R_{12}^{\prime}=R_{12}+\Delta R_{12}$ with

$$
\Delta T_{12}=-\Delta R_{12}=\frac{4\left(n_{2}-n_{1}\right)}{\left(n_{1}+n_{2}\right)^{3}}\left(n_{2} \Delta n_{1}-n_{1} \Delta n_{2}\right)
$$

The central and band edge wavelength changes $\Delta \lambda_{0}$ and $\Delta \lambda_{B}$ are then related through the relation

$$
\frac{\Delta \lambda_{B}}{\lambda_{B}}=4 \frac{n_{1}-n_{2}}{\left(n_{1}+n_{2}\right)^{3}}\left(n_{2} \Delta n_{1}-n_{1} \Delta n_{2}\right) \frac{1+\cos (\pi / N)}{\pi \frac{\lambda_{0}}{\lambda_{B}} \sin \left(\pi \lambda_{0} / \lambda_{B}\right)}+\frac{\Delta \lambda_{0}}{\lambda_{0}}
$$

For normal incidence the central wavelength of the forbidden band is given by $\lambda_{0} / 2=n_{1} e_{1}+n_{2} e_{2}$, so that 


$$
\frac{\Delta \lambda_{0}}{\lambda_{0}}=\frac{1}{2}\left(\frac{\Delta n_{1}}{n_{1}}+\frac{\Delta n_{2}}{n_{2}}\right)
$$

Equations I.6 and 7 entirely relate the band edge shift to the refractive index changes.

\section{Experimental setup}

The band edge shift at high intensities was measured by recording around the band edge wavelength the reflectivity spectra of both pump and probe beams at various pump intensities. Such experiments allow both the determination of the very high refractive index changes occurring at the band edge of the photonic crystal and the physical origin of the nonlinearity. Figure 1 shows the schematic experimental setup. Pump and probe beams originate from the same optical parametric generator pumped by a frequency doubled modelocked Nd-YAG laser [19]. They are both focused onto the sample, the pump beam orthogonally to the sample, the probe beam with an incidence angle of few degrees. Pump and probe reflectivity are determined thanks to the four photodiodes PD1 to PD4 connected to a boxcar and computer acquisition system. Finally, the delay line allows the variation of the pump-probe delay for time resolved experiments.

In order to clarify the role played by the index contrast and the number of periods in the enhancement of the local field factor and evaluate the ability of these devices for optical signal processing, two different photonic crystals were tested: a 30 pair thick $\mathrm{Cd}_{0.75} \mathrm{Mn}_{0.25} \mathrm{Te} / \mathrm{Cd}_{0.40} \mathrm{Mg}_{0.60} \mathrm{Te}$ photonic crystal exhibiting a low refractive index contrast $\left(n_{1}-n_{2}=0.50\right)$ and a high index contrast $\left(n_{1}-n_{2}=1.71\right) \mathrm{Al}_{0.30} \mathrm{Ga}_{0.70} \mathrm{As} / \mathrm{AlOx}$ photonic crystal with 10 pairs of layers. As the wafer was absorbing experiments were performed in reflexion on a Bragg mirror deposited on this wafer before the growth of the photonic crystal of interest [13]. The device is shown in Figure 2.a in the case of the III-V photonic crystal. The thicknesses of the layers of the Bragg mirror were fixed in order to have the central 
wavelength of this Bragg mirror equal to the band edge wavelength $\lambda_{B}$ of the photonic crystal. Figures 2.b, c, and d present the calculated reflectivity spectra of the photonic crystal alone, that of the Bragg mirror and that of the total device respectively in the case of scattering losses of $0.07 \%$ at each interface. These spectra obtained for a band edge located around 1500 $\mathrm{nm}$ were calculated to illustrate the general behaviour of the device. The dip of reflectivity of the device present at the band edge is a result of light localization which strongly enhances the scattering losses of the total device $[11,12]$. In particular, only a very small dip due to the finite thickness of the Bragg mirror would appear without scattering, which indicates that this kind of structures could also be very useful for measuring losses in photonic crystals. Table 1 which summarizes the parameters of the two samples shows the major differences between the two photonic crystals: the II-VI semiconductor PC $\left(\mathrm{Cd}_{0.75} \mathrm{Mn}_{0.25} \mathrm{Te} / \mathrm{Cd}_{0.40} \mathrm{Mg}_{0.60} \mathrm{Te}\right)$ is thick with 30 pairs of layers of low refractive index contrast while a thinner III-V semiconductor PC $\left(\mathrm{Al}_{0.30} \mathrm{Ga}_{0.70} \mathrm{As} / \mathrm{AlOx}\right)$ of high refractive index contrast is also considered in our experiments.

\section{Nonlinear Reflexion Spectroscopy}

Nonlinear reflectivity spectra of the pump beam and probe reflectivity modifications induced by the pump beam for synchronized pump and probe pulses were recorded in the two cases of II-VI and III-V semiconductor photonic crystals.

\section{Nonlinear Reflexion Spectroscopy in $C d_{0.75} M n_{0.25} T e / C d_{0.40} M g_{0.60} T e$}

Spectra were recorded for the pump beam set at normal incidence and for a probe beam having an incidence angle of 6 degrees. Figure 3 shows the reflectivity spectra of the pump beam of intensity $0.64 \mathrm{GW} / \mathrm{cm}^{2}$ (full triangle) and that of the probe beam in the absence (open circle) and in the presence (full circle) of the pump beam. A large blue shift of $1.5 \mathrm{~nm}$ is observed for the probe reflectivity at this high pump intensity. Although smaller this shift also 
exists for the pump beam. In fact several spectra were recorded at different pump intensities. The corresponding blue shifts $\Delta \lambda$ are plotted in figure 4 using squares and circles for pump and probe beams respectively. An almost perfect linear dependence of $\Delta \lambda$ with respect to the pump intensity is observed in both cases. The scale is given in $\mathrm{nm}$ for the observed blue shifts (left part of the figure). The vertical axis also represents the induced refractive index changes (right part of the figure) deduced from equations I.6 and 7. The correspondence was determined by assuming that the index changes are significant only in $\mathrm{Cd}_{0.75} \mathrm{Mn}_{0.25} \mathrm{Te}$ (due to the proximity of the electronic band gap resonance for the wavelengths considered (around $692 \mathrm{~nm})$ and negligible $\left(\Delta n_{2}=0\right)$ for the large band gap $\mathrm{Cd}_{0.40} \mathrm{Mg}_{0.60}$ Te semiconductor. Moreover, the slope for the probe beam is twice that corresponding to the pump beam within a precision of only $3 \%$. This remarkable result is very well explained if we remember that, due to different degeneracy factors, the nonlinear susceptibility is twice larger for crossed Kerr effect than for self Kerr interaction [21].

\section{Nonlinear Reflexion Spectroscopy in $A I_{0.30} G a_{0.70} A s / A I O x$}

Spectra were recorded for the pump beam set at normal incidence and for a probe beam having an incidence angle of 4 degrees. Figure 5 shows the reflectivity spectra of the probe beam in the presence of a $1.3 \mathrm{GW} / \mathrm{cm}^{2}$ intensity pump beam (full circles) and in the linear regime with no pump beam(full squares). A very large blue shift of $7.5 \mathrm{~nm}$ is observed at this high pump intensity. Assuming that the index changes are significant only in $\mathrm{Al}_{0.30} \mathrm{Ga}_{0.70} \mathrm{As}$ and negligible $\left(\Delta n_{2}=0\right)$ for $\mathrm{AlOx}$ which is a very bad nonlinear medium, a refractive index change $\Delta n_{1}=-0.019$ was deduced from the blue shift of the band gap. Note that this value is three times larger than that measured in II-VI semiconductor PC although the laser wavelength was much less resonant. 
As discussed in section IV, the very strong refractive index changes measured in both experiments demonstrate the major role played by the nano-structuration of the semiconductor material in increasing the nonlinear susceptibilities.

\section{Time Resolved Experiments : Physical Origin of the Nonlinearities}

Time resolved experiments were also undertaken in both II-VI and III-V samples in order to identify the physical origin of the optical nonlinearities, a peculiarity of major importance in applications since it governs the response time of a device.

\section{Time resolved experiments in $C d_{0.75} M n_{0.25} T e / C d_{0.40} M g_{0.60} T e$}

The nonlinear probe reflectivity in the presence of the pump pulse was measured at the edge of the minimum of reflectivity $(\lambda=693.5 \mathrm{~nm})$ and compared to the linear reflectivity for various pump-probe delays. The zero delay was determined by measuring the conjugate intensity due to the four-wave mixing process occurring in the sample due to the counterpropagating pump beams and the probe beam [13]. Figure 6 shows this conjugate intensity normalized to that of the pump and probe beams (closed squares) together with the ratio of the nonlinear and linear reflectivity (closed circles), both plotted as a function of the pump-probe delay.

Assuming the same Gaussian shape for the pump and probe pulses which are issued from the same laser $\left(I_{P} \propto I_{S} \propto \exp \left(-t^{2} / \Delta t^{2}\right)\right.$ ), the delay dependant conjugate energy measured by PD4 writes

$$
E_{C}(\tau) \propto \int_{-\infty}^{+\infty} I_{P}{ }^{2}(t) I_{S}(t+\tau) d t \propto \exp \left(\frac{-\tau^{2}}{(\sqrt{3 / 2} \cdot \Delta t)^{2}}\right)
$$

The use of equation III.1 and figure 6 gives the value $2 \sqrt{\operatorname{Ln}(2)} \Delta t=13.3 \mathrm{ps}$ for the full width at half maximum (FWHM) laser pulse duration in good agreement with second harmonic generation autocorrelation measurements. The centre of this four-wave mixing autocorrelation 
pulse provides the zero delay. As shown in figure 6 this zero delay corresponds to half the maximum of the reflectivity change $\mathrm{R}_{\mathrm{NL}} / \mathrm{R}_{\mathrm{L}}$ with $\mathrm{R}_{\mathrm{NL}}$ and $\mathrm{R}_{\mathrm{L}}$ the probe reflectivities with the pump beam present and in the linear regime respectively. This demonstrates that no significant instantaneous process occurs in this sample and that the refractive index changes responsible for the blue shift of the PC band edge can be safely attributed to free carriers generated in $\mathrm{Cd}_{0.75} \mathrm{Mn}_{0.25} \mathrm{Te}$ by two-photon absorption. Figure 6 showing the time evolution of the ratio of the nonlinear $\left(\mathrm{R}_{\mathrm{NL}}\right)$ and linear $\left(\mathrm{R}_{\mathrm{L}}\right)$ probe reflectivity also demonstrates that the free carrier relaxation is long (probably more than $1 \mathrm{~ns}$ ) since the band edge blue shift remains almost unchanged during the first 100 ps following the pump excitation. This point could be detrimental to such PCs for the purpose of fast optical signal processing.

\section{Time resolved experiments in $A l_{0.30} G a_{0.70} A s / A I O x$}

As in the case of the II-VI sample, the nonlinear probe reflectivity in the presence of the pump pulse was measured at the edge of the minimum of reflectivity $(\lambda=1480.4 \mathrm{~nm})$ and compared to the linear reflectivity for various pump-probe delays. However, due to a strong scattering of the pump beam, the zero delay could not be determined using the four-mixing process. We use interferences between the reflected probe beam and the pump beam scattering to determine this zero delay. A mini-shaker applied to one of the mirrors inserted in the optical path of the pump beam allowed the measurement of the contrast of the interferences between the reflected probe beam and the pump scattering. This measurement is directly related to the cross-correlation function of the pump and probe beam. This cross-correlation function is plotted in figure 7 using closed circles. The continuous line is a fit obtained using a Gaussian shape for the initial laser pulses. The corresponding FWHM pulse duration is $14 \mathrm{ps,} \mathrm{in} \mathrm{good}$ agreement with measurements performed at $694 \mathrm{~nm}$. The centre of this cross-correlation pulse provides the zero delay (the maximum of this pulse is less than unity probably due to residual speckle). Figure 7 also shows the ratio of the nonlinear and linear reflectivities plotted on the 
same scale (using closed squares). It appears from this figure that an instantaneous Kerr induced contribution is superimposed to the long-lived free carrier contribution. The refractive index changes $\Delta n_{K}=-0.013$ and $\Delta n_{F C}=-0.012$ were deduced from figure 7 for Kerr and free carriers contributions respectively. It is important to note that even for a laser frequency below the half band gap free carriers were photo-generated through multi-photon absorption (three-photon absorption in this case). In view of very fast devices, this means that the PCs must be optimized for an operation at lower pump intensities in order to avoid the long lived free carrier contribution.

\section{Discussion}

\section{The local field factor}

At the band edge of the PC, if the linear and nonlinear polarizations are written as a function of the fields outside of the PC, the effective linear and $\mathrm{n}^{\text {th }}$ order nonlinear susceptibilities $\chi_{\text {eff }}^{(1)}$ and $\chi_{\text {eff }}^{(n)}$ respectively can be given by $[11,12]$

$$
\begin{aligned}
& \chi_{e f f}^{(1)}=f \chi_{0}^{(1)} \\
& \chi_{e f f}^{(n)}=f^{n+1} A_{n}^{p} \chi_{0}^{(n)}
\end{aligned}
$$

Where $\chi_{0}^{(1)}$ and $\chi_{0}^{(n)}$ are the linear and fully degenerate $\mathrm{n}^{\text {th }}$ order nonlinear susceptibilities of the bulk material. In equations IV.1.a and $\mathrm{b}, A_{n}^{p}=n ! / p$ ! is the degeneracy factor for $\mathrm{p}$ identical waves in the nonlinear interaction [20] and $f=\left|E_{\text {in }} / E_{\text {out }}\right|$ is the local field factor which relates the enhancement of the field $E_{\text {in }}$ inside the PC when compared to the same field outside the PC $\left(E_{\text {out }}\right)$. The enhancement occurs at the band edge of the PC thanks to multiple interferences as it is the case in Fabry-Perot cavities. Evidently, Eq. IV.1.b shows 
that high order nonlinear processes are more efficient in PCs than in bulk materials and that the effect of light localization is stronger for higher order nonlinear interactions.

In the CdMnTe/CdMgTe photonic crystal, the value $\mathrm{f}=4.2$ was derived from the result of the experiments for the local field factor [13]. In the case of GaAlAs/AlOx, the instantaneous crossed-Kerr refractive index change was related to the effective third order nonlinear susceptibility through the relation [20]

$$
\chi_{\text {eff }}^{(3)}=\frac{c f n_{0}^{2} \Delta n_{K}}{4 \pi^{2} I}
$$

with $n_{0}=\sqrt{n_{1} n_{2}}$ the mean refractive index of the photonic crystal. Combining equations IV.1.b and IV.2 and using $\Delta n_{K}=-0.013$ for $I=1.3 \mathrm{GW} / \mathrm{cm}^{2}$ and $6 \chi_{0}^{(3)}=-8.0 \times 10^{-11}$ esu (measured in bulk GaAlAs through totally non degenerate four wave mixing [21]), one gets $f$ $=3.7$. Note that this value is in close agreement with that $f=4$ calculated using ref. 12 .

Comparing the values of the local field factors in both PCs shows that about the same value of 4 is obtained in both cases although the number of pairs is three times smaller in the large index contrast GaAlAs/A1Ox PC. This result indicates that, although they are more difficult to grow, large index contrast PC's (GaAlAs/AlOx or even better semiconductor/air) are preferable for small volume optical processing.

\section{Free carrier generation}

At low intensities the semiconductor materials are transparent at the wavelengths used in the experiments. However, at the high pump intensities needed for large shifts of the optical band edge of the 1D-PC, multi-photon absorption processes occur. Moreover, at the photonic band edge wavelength the effective nonlinear susceptibilities responsible for multi-photon absorption are enhanced by factors $\mathrm{f}^{4}$ and $\mathrm{f}^{6}$ for two- and three-photon absorption respectively. This gives rise to generation of free carriers in the semiconductor medium, with consequences for the value of the refractive index (see section III). 
In the case of the CdMnTe/CdMgTe photonic crystal the optical band edge frequency $(\omega=1.79 \mathrm{eV})$ is smaller than the electronic band gap $\left(\omega_{\mathrm{G}}=2.00 \mathrm{eV}\right)$ of CdMnTe, which makes the semiconductor medium transparent in the linear regime. On the other hand, $\omega>\omega_{G} / 2$ and two-photon absorption occurs. Free carriers are then generated with a density

$$
N_{C}=\frac{1}{2} \frac{\lambda}{h c} \frac{\Delta W}{S L}
$$

Where the factor 2 has been introduced since two photons are needed for creating one electron-hole pair. In equation IV.3 $\Delta W$ is the absorbed energy, $L=30 \lambda / 4 / n_{1}=1.6 \mu \mathrm{m}$ the total thickness of the CdMnTe layers, and $S=0.031 \mathrm{~mm}^{2}$ the laser beam cross-section. For small absorption of the pump pulse of energy $W=2.7 \mu \mathrm{J}, \Delta W=f^{4} \alpha_{2} I W L$ with $\alpha_{2}=1.7 \mathrm{GW}^{-}$ ${ }^{1} \mathrm{~cm}$ the bulk two-photon absorption coefficient of CdMnTe [22] and $I=W /(S \Delta t)$ the pump beam intensity, so that the carrier density amounts up to $N_{C}=5.3 \times 10^{18} \mathrm{~cm}^{-3}$ for a small absorption $\Delta W / W$ of only $5 \%$. This high carrier density obtained at relatively low pump fluence $\left(8.7 \mathrm{~mJ} / \mathrm{cm}^{2}\right)$ is evidently due to the strong enhancement of the two-photon absorption process by the photonic crystal.

In the case of the GaAlAs/AlOx photonic crystal the optical band edge frequency $(\omega=$ $0.848 \mathrm{eV})$ is smaller than half the electronic band gap of GaAlAs $\left(\omega_{\mathrm{G}}=1.96 \mathrm{eV}\right)$, so that twophoton absorption does not occur. Following the same approach as in the case of the II-VI PC, free carriers are found to be photo-generated by three-photon absorption with a density

$$
N_{C}=\frac{1}{3} \frac{\lambda}{h c} f^{6} \alpha_{3} I^{3} \Delta t
$$

Where $\alpha_{3}=0.03 \mathrm{GW}^{-2} \mathrm{~cm}^{3}$ is the nonlinear coefficient for three-photon absorption in GaAlAs [23]. For the pump pulse of peak intensity $I=1.3 \mathrm{GW} / \mathrm{cm}^{2}$ and FWHM duration $\Delta t=14 \mathrm{ps,}$ the carrier density amounts up to $N_{C}=5.9 \times 10^{18} \mathrm{~cm}^{-3}$. Note that this value is about the same as that measured in the case of two-photon absorption. This means that because of the 
enhancement of three-photon absorption (by a factor $f^{6}$ ) free carriers are efficiently produced even for a pump frequency smaller than $\omega_{G} / 2$, when two-photon absorption cannot occur.

\section{Towards devices for optical signal processing?}

In order to discuss the potentiality of $1 \mathrm{D}$ photonic crystals to dense optical signal processing the main results obtained for both PCs are summarized in Table 2 together with the structural characteristics of these samples (refractive index contrast and number of pairs of layers). For the purpose of clear comparison, the quantities listed in the three last columns correspond to the same pump intensity of $1 \mathrm{GW} / \mathrm{cm}^{2}$ for both PCs.

It should be first noticed that the local field factors are about the same $(\sim 4)$ although the III-V sample is thinner than the II-VI one (three times less pairs of layers). This demonstrates that high contrast PCs have a high potentiality for low volume devices for optical signal processing.

From table 2 it also appears that, at the same pump intensity of $1 \mathrm{GW} / \mathrm{cm}^{2}$, the blue shift of the band edge is larger in III-V than in II-VI photonics crystals. This large blue shift is mainly due to pure Kerr effect, the contribution of which is twice larger than that due to free carriers. These two examples also show that free carriers are photo-generated in PCs at large pump intensities whatever two- or three-photon absorption is concerned, with densities only five times smaller for the later. For low speed optical processing, two-photon absorption is favourable for large refractive index changes $(\Delta n=0.016)$ and II-VI PCs may be used. On the contrary, for the purpose of applications to fast optical information processing where high repetition rate devices are necessary, free carriers must be avoided because they limit their response time and, probably more important, because they are responsible for undesirable thermal heating. In view of these properties, two-photon absorption must be strictly avoided. This is the case in the III-V sample which exhibits a Kerr induced index change twice larger than that due to free carriers, whereas only the free carrier contribution appears in the II-VI 
PC. However, the free carrier density measured at $1 \mathrm{GW} / \mathrm{cm}^{2}$ in the III-V PC is still too large for potential applications. Working at a lower intensity $\left(0.2 \mathrm{GW} / \mathrm{cm}^{2}\right.$ for instance $)$ would limit the carrier density down to $2 \times 10^{16} \mathrm{~cm}^{-3}$ and the corresponding refractive index change to $1.7 \times 10^{-6}$, a value which is much lower than that due Kerr effect $\left(2.0 \times 10^{-3}\right.$ at the same pump intensity). Due to the large bandwidth of the band edge in our sample such an intensity is currently too small for high contrast reflectivity changes in presence of the pump beam. However, models predict narrower resonances for the band edge [12]. Therefore, if a well controlled fabrication of the PC is realized, very small devices driven by low power pump pulses are possible with fast response times and almost no heating of the device.

\section{Conclusion}

Time resolved nonlinear spectroscopy recorded at the band edge of $1 \mathrm{D}$ photonic crystals showed that very large refractive index changes were possible both in very thin high index contrast and in thicker low index contrast photonic crystals. These very large values obtained at moderate pump power have been shown to occur for laser wavelengths close to the edge of the photonic band gap of the 1D-PC due the strong optical nonlinearities induced by the high local intensity existing inside the PC at these wavelengths. Such a property makes these very small devices very attractive for the purpose of optical signal processing. The study has also demonstrated photo-generation of high free carrier densities through two- or even three-photon absorption processes which are also strongly enhanced by the local field factor at the band edge of the photonic crystal. As the free carrier lifetime can intrinsically limit the response time of the device, this drawback must be circumvented for future application of the technique. This could be made possible by using lower pump intensities in photonic crystals showing narrower resonances. 


\section{References}

1. J. M. Lourtioz, H. Benisty, V. Berger, J.M. Gérard, D. Maystre, A. Tchelnelkov. "Photonic Crystals", Springer-Verlag, Berlin, Heidelberg (2008)

2. M. Bertolotti. "Wave interactions in photonic band structures: an overview" J. Opt. A: Pure Appl. Opt. 8, S9-S32 (2006)

3. Y. Dumeige, I. Sagnes, P. Monnier, P. Vidakovic, C. Meriadec, A. Levenson " $\chi^{(2)}$ semiconductor photonic crystals" J. Opt. Soc. Am. B 19, 2094-2101 (2002)

4. A. V. Andreev, A. V. Balakin, A. B. Kozlov, I. A. Ozheredov, I. R. Prudnikov, A. P. Shkurinov, P. Masselin, G. Mouret, "Four-wave mixing in one-dimensional photonic crystals: inhomogeneous-wave excitation" J. Opt. Soc. Am. B 19, 1865-1872 (2002)

5. F. Wang and S. N. Zhua, K. F. Li, K. W. Cheah "Third-harmonic generation in a one-dimensional photonic-crystal-based amorphous nanocavity" Appl. Phys. Lett. 88, $071102(2006)$

6. C. Becker, M. Wegener, S. Wong, G. von Freymann. "Phase-matched nondegenerate four-wave mixing in one-dimensional photonic crystals" Appl. Phys. Lett. 89, 131122 (2006).

7. Weixin Lu, Ping Xie, Zhao-Qing Zhang, George K. L. Wong, Kam Sing Wong "Simultaneous perfect phase matching for second and third harmonic generations in ZnS/YF3 photonic crystal for visible emissions" Opt. Express 14, 12353 (2006)

8. F. Benabid "Hollow-core photonic bandgap fibre: new light guidance for new science and technology" Phil. Trans. R. Soc. A 364, 3439 (2006)

9. S. Lebrun, Ph. Delaye, R. Frey, G. Roosen. "High-efficiency single-mode Raman generation in a liquid-filled photonic bandgap fiber". Opt. Lett. 32, 337 (2007).

10. F. Raineri, Crina Cojocaru, P. Monnier, A. Levenson, and R. Raj and C. Seassal, X. Letartre, and P. Viktorovitch, "Ultrafast dynamics of the third-order nonlinear response in a two-dimensional InP-based photonic crystal”, Appl. Phys. Lett. 85, 1880, (2004)

11. R. Frey, Ph. Delaye, G. Roosen, "Third order optical nonlinearities in photonic crystals", Nanophotonics, H. Rigneault, J.M. Lourtioz, C. Delalande, A. Levenson eds. ISTE p.187-201 (2006)

12. Ph. Delaye, M. Astic, R. Frey, G. Roosen, "Transfer-matrix modeling of four-wave mixing at the band edge of a one-dimensional photonic crystal", J. Opt. Soc. Am. B 22, 2494 (2005) 
13. L. Razzari, D. Träger, M. Astic, Ph. Delaye, R. Frey, G. Roosen, R. André, "Kerr and four-wave mixing spectroscopy at the band edge of one-dimensional photonic crystals", Appl. Phys. Lett. 86, 231106 (2005).

14. Jie Shen, Zhuangjian Zhang, Zhongyi Hua, Guohong Ma, Sing Hai Tang "Observation of two-photon absorption enhancement at double defect modes in onedimensionnal photonic crystals" Appl. Phys. Lett. 88, 011113 (2006)

15. Ph. J. Harding, T. G. Euser, Y. R. Nowicki-Bringuier, J.M. Gérard, W. L. Vos, "Dynamical ultrafast all-optical switching of planar GaAs/AlAs photonic microcavities" Appl. Phys. Lett. 91, 111103 (2007).

16. Geon Joon Lee, Young Pak Lee, Sung Goo Jung, Chang Kwon Hwangbo, Sunman Kim, Inkyu Park "Linear and nonlinear optical properties of one-dimensional photonic crystals containing ZnO defects" J. Appl. Phys. 102, 073528 (2007)

17. Jisoo Hwang, Min Jung Kim, J. W. Wu, Seung Mook Lee, Bum Ku Rhee. "Picosecond pump-probe measurement of bandgap changes in $\mathrm{SiO} 2 / \mathrm{TiO} 2$ onedimensional photonic bandgap structures" Opt. Lett. 31, 377 (2006).

18. J.M. Bendickson, J.P. Dowling, and M. Scalora, 'Analytic expressions for the electromagnetic mode density in finite, one dimensional, photonic band-gap structures', Physical Revue.E 53, 4107-4121 (1996)

19. R. Pankoke, A. Cassinelli, P. Sillard, C. Dorrer, P. Leisching et R. Frey, 'Générateurs et amplificateurs paramétriques optiques monomode transverse', Annales de Physique, 20, 593 (1995)

20. R.W. Boyd, Nonlinear Optics, Academic Press, New York, 2003

21. H.Q. Le, D.E. Bossi, K.B. Nichols, W.D. Goodhue, 'Observation of maker fringes and estimation of $\chi^{(3)}$ using picosecond nondegenerate four-wave-mixing in AlGaAs waveguides', Applied Physics Letters, 56, 1008-1010 (1989). H.Q. Le, S. Di Cecca, 'Ultrafast, romm temperature, resonance-enhanced third-order optical susceptibility tensor of an AlGaAs/GaAs quantum well', Optics Letters, 16, 901-903 (1991)

22. Serge Hugonnard-Bruyère, Ph. D Thesis, Ecole Polytechnique (1994)

23. J.S. Aitchison, D.C. Hutchings, J.U. Kang, G.I. Stegeman, A. Villeneuve, 'The non linear optical properties of AlGaAs at the half band gap', IEEE Journal of Quantum Electronics, 33, 341 (1997) 


\section{Table captions}

Table 1. Characteristics of the II-VI and III-V photonic crystals.

Table 2. Performances of the II-VI and III-V photonic crystals 


\section{Figure Captions}

Figure 1. Schematic diagram of the experimental setup. PDi are photodiodes for energy measurements of the incident $(i=1$ and 3$)$ and reflected $(i=2$ and 4$)$ pump and probe beams.

Figure 2. Scheme and photograph of the III-V Sample (a) and, reflectivity spectra of the studied photonic crystal alone (b), the Bragg mirror alone (c) and the total device (d).

Figure 3. II-VI PC reflectivity spectrum for the pump beam (closed triangle) and for the probe beam with and without the pump beam present (closed and open circles respectively).

Figure 4. II-VI PC band edge wavelength shift and associated refractive index change plotted as a function of the pump intensity for the pump and probe beams (closed squares and circles respectively).

Figure 5. III-V PC reflectivity spectrum for the probe beam with and without the pump beam present (closed circles and squares respectively).

Figure 6. II-VI four-wave mixing autocorrelation intensity and ratio of the nonlinear and linear reflectivity of the probe beam plotted as a function of the pump-probe delay.

Figure 7. III-V interferometric autocorrelation intensity and ratio of the nonlinear and linear reflectivity of the probe beam plotted as a function of the pump-probe delay. 
Tables

\begin{tabular}{|c|c|c|c|c|c|c|c|}
\hline type & $\begin{array}{c}\text { Layer 1 } \\
\text { Material }\end{array}$ & $e_{1}(\mathrm{~nm})$ & $n_{1}$ & $\begin{array}{c}\text { Layer 2 } \\
\text { Material }\end{array}$ & $e_{2}(\mathrm{~nm})$ & $n_{2}$ & $\begin{array}{c}\text { Pair } \\
\text { Number }\end{array}$ \\
\hline II-VI & $\mathrm{Cd}_{0.75} \mathrm{Mn}_{0.25} \mathrm{Te}$ & 54 & 3.088 & $\mathrm{Cd}_{0.40} \mathrm{Mg}_{0.60} \mathrm{Te}$ & 64.6 & 2.585 & 30 \\
\hline $\mathrm{III}-\mathrm{V}$ & $\mathrm{Al}_{0.30} \mathrm{Ga}_{0.70} \mathrm{As}$ & 87.9 & 3.323 & $\mathrm{AlOx}$ & 180.9 & 1.6 & 10 \\
\hline
\end{tabular}

Table I

\begin{tabular}{|c|c|c|c|c|c|c|c|}
\hline type & $\begin{array}{c}\text { Index } \\
\text { Constrast }\end{array}$ & $\begin{array}{c}\text { Pair } \\
\text { Number }\end{array}$ & $f$ & $\begin{array}{c}\Delta \lambda_{B}(\mathrm{~nm}) \\
@ \\
1 \mathrm{GW} / \mathrm{cm}^{2}\end{array}$ & $\begin{array}{c}\text { Physical Origin } \\
\Delta n\end{array}$ & $\begin{array}{c}\text { Free carrier } \\
\text { density } \\
\left(\mathrm{cm}^{-3}\right)\end{array}$ \\
\hline II-VI & 0.50 & 30 & 4.2 & 3.7 & \multicolumn{2}{|c|}{$\begin{array}{c}\text { Free Carrier } \\
-0.016\end{array}$} & $13 \times 10^{18}$ \\
\hline III-V & 1.71 & 10 & 3.7 & 5.9 & $\begin{array}{c}\text { Free Carrier } \\
-0.005\end{array}$ & $\begin{array}{c}\text { Kerr } \\
-0.010\end{array}$ & $2.7 \times 10^{18}$ \\
\hline
\end{tabular}

Table II 
Figures

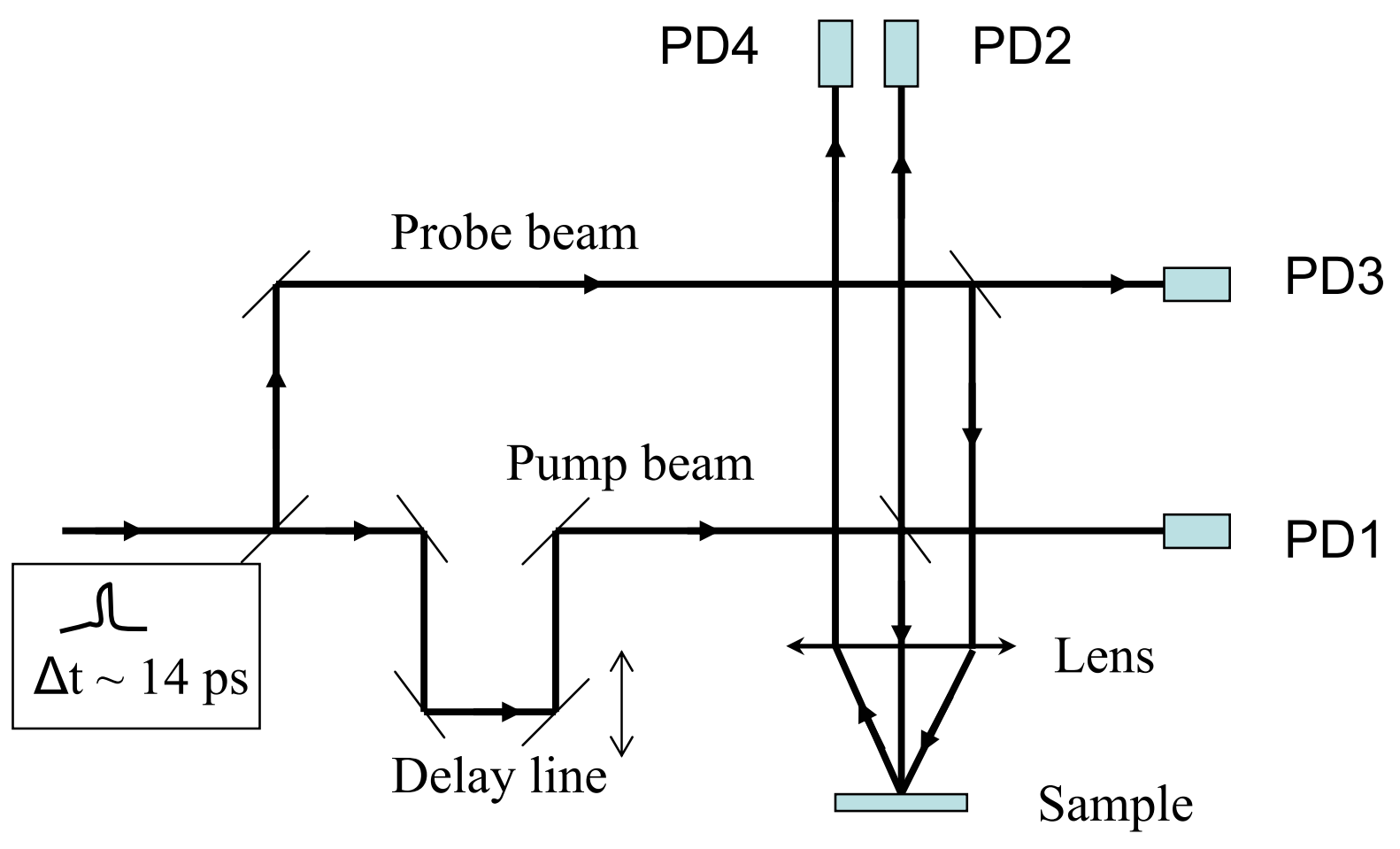

Figure 1 
(a)

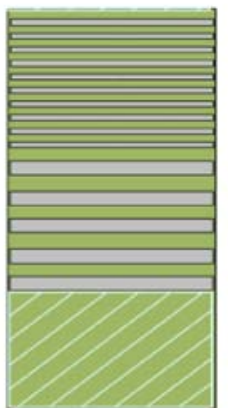

1D-PC :

10 pairs of layers

$\mathrm{Al}_{0.30} \mathrm{Ga}_{0.70} \mathrm{As} / \mathrm{AlOx}$

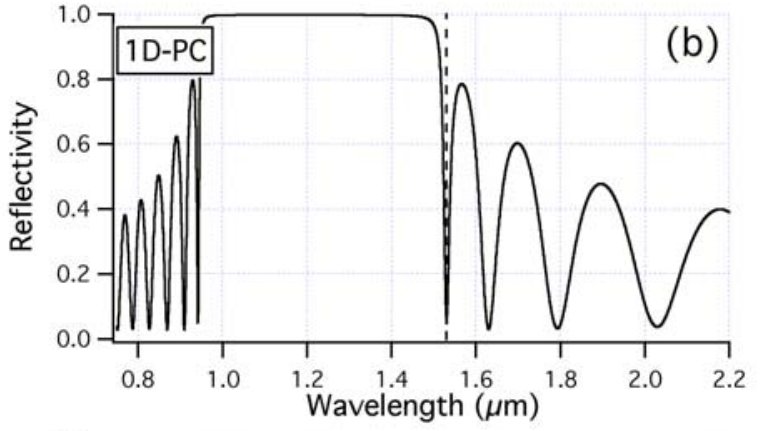

Bragg :

5 pairs of layers

$\mathrm{Al}_{0.30} \mathrm{Ga}_{0.70} \mathrm{As} / \mathrm{AlOx}$
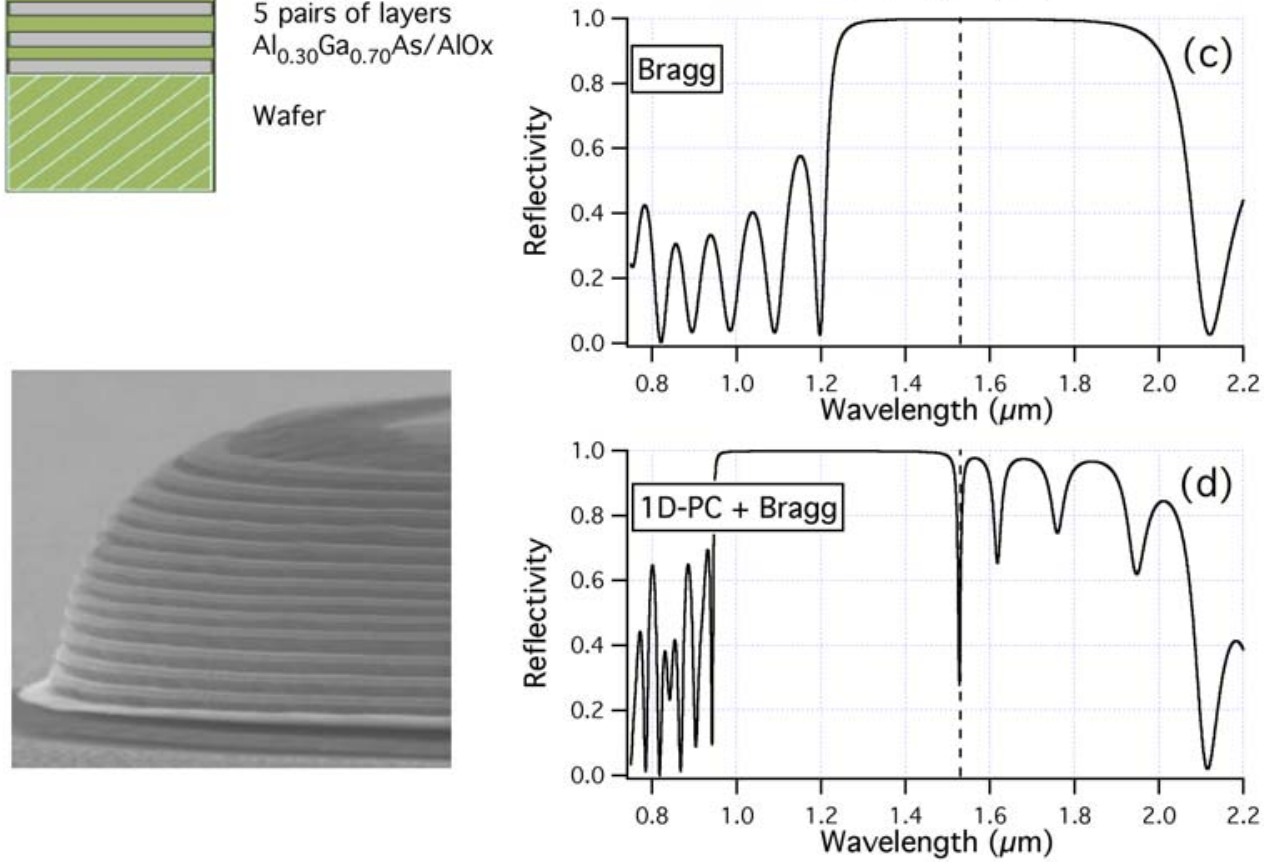

Figure 2 


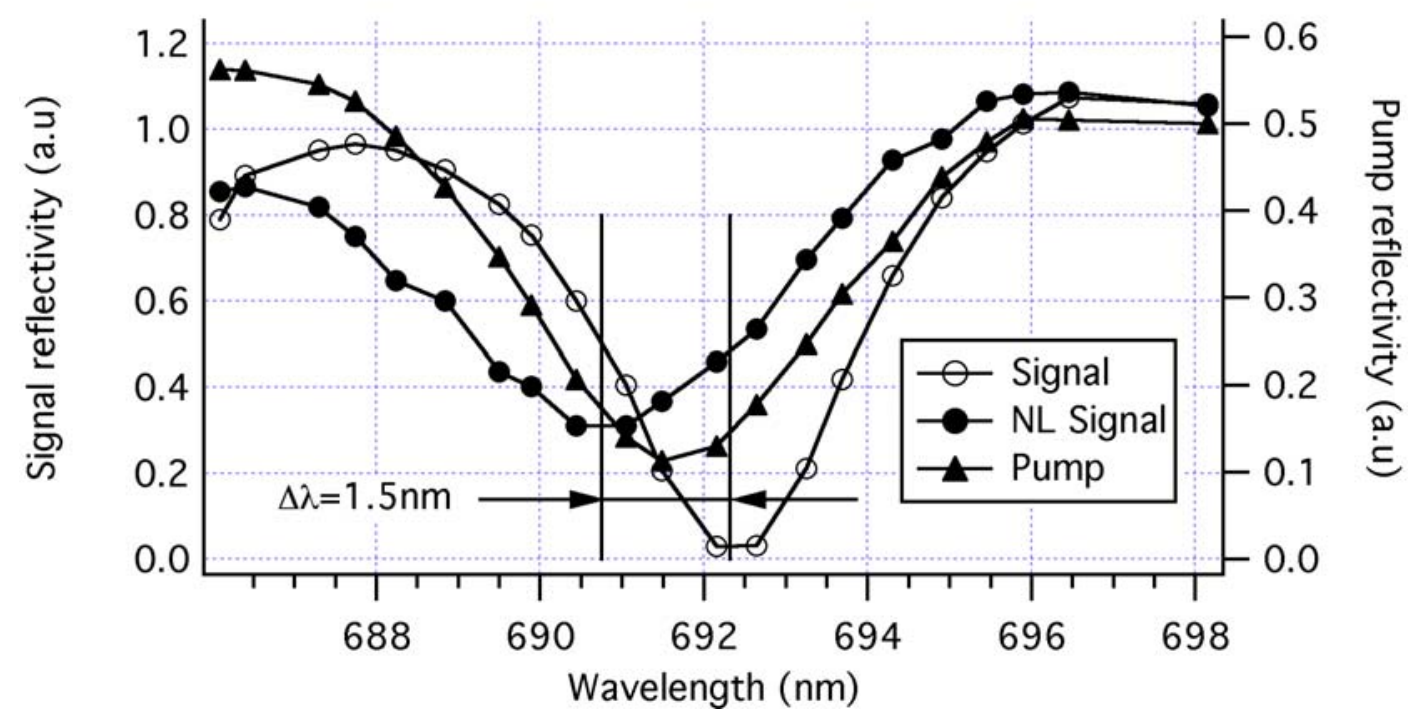

Figure 3 


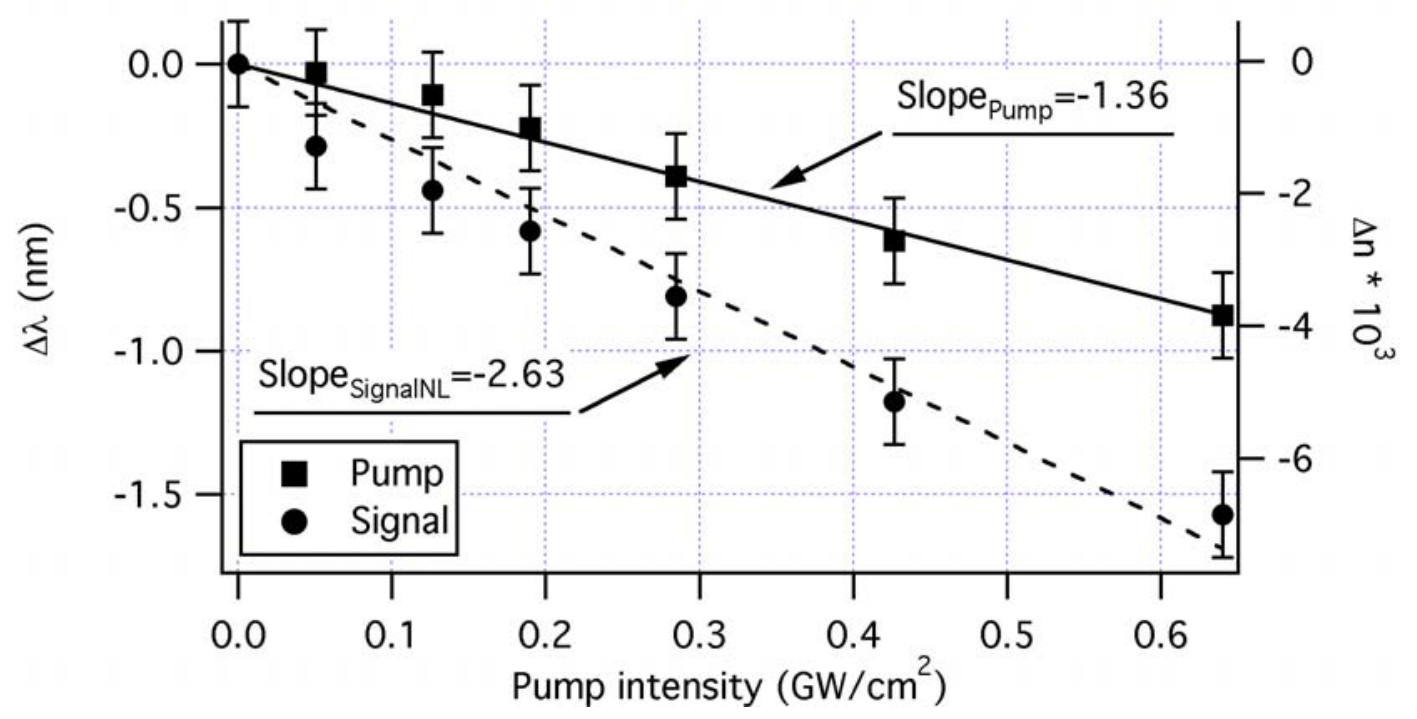

Figure 4 


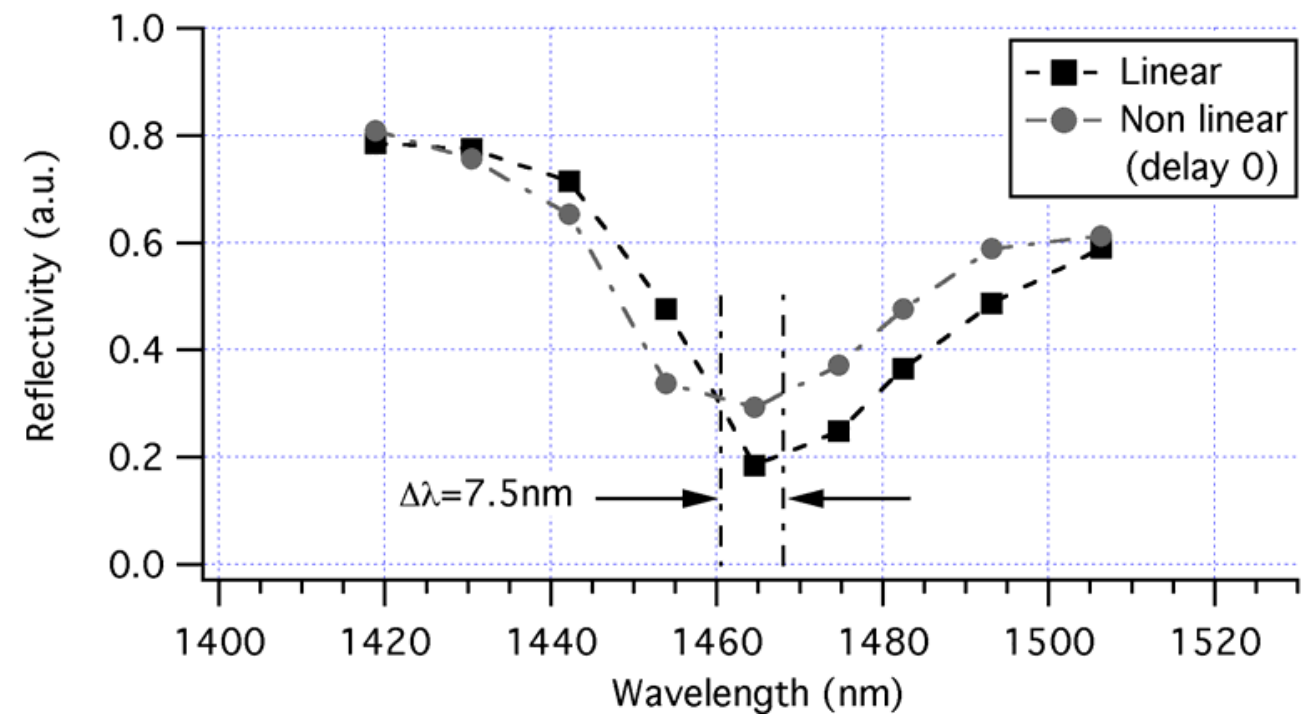

Figure 5 


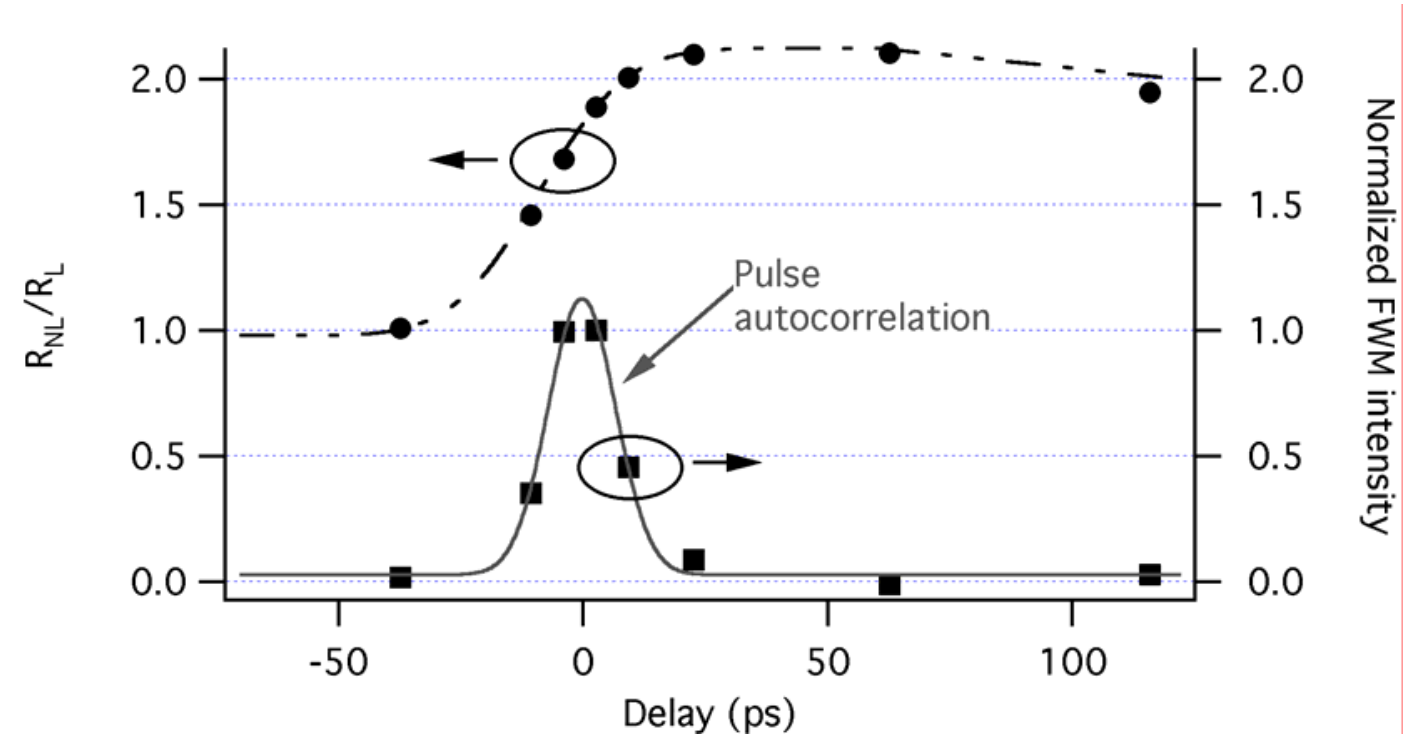

Figure 6 


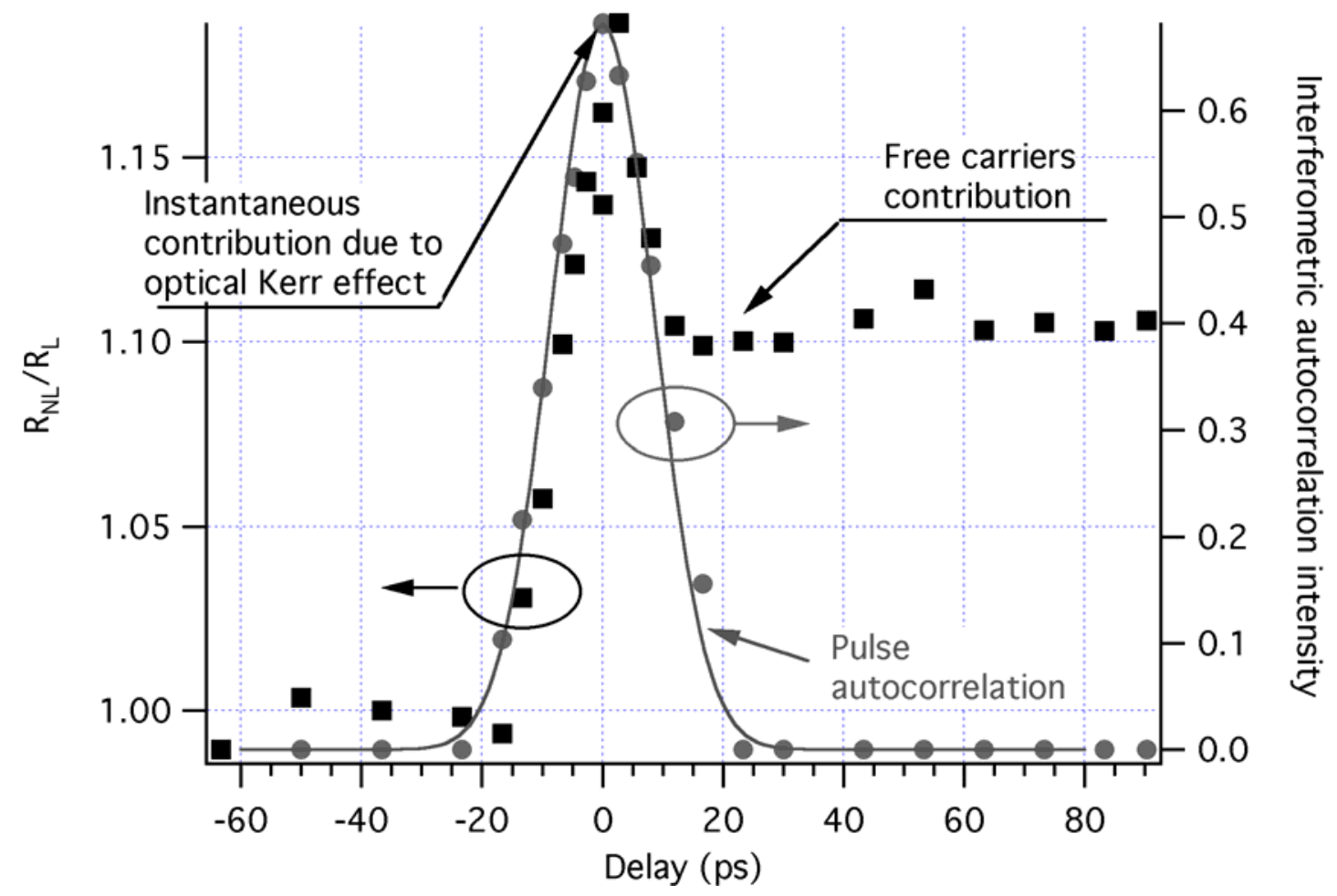

Figure 7 